\title{
Validity of an algorithm to identify osteonecrosis of the jaw in women with postmenopausal osteoporosis in the Danish National Registry of Patients
}

\author{
This article was published in the following Dove Press journal: \\ Clinical Epidemiology \\ 31 July 2013 \\ Number of times this article has been viewed
}

\author{
Henrik Gammelager' \\ Claus Sværke' \\ Sven Erik Noerholt ${ }^{2}$ \\ Bjarne Neumann-Jensen ${ }^{3}$ \\ Fei Xue ${ }^{4}$ \\ Cathy Critchlow ${ }^{4}$ \\ Johan Bergdahl ${ }^{5}$ \\ Ylva Trolle Lagerros ${ }^{5}$ \\ Helle Kieler ${ }^{5}$ \\ Grethe S Tell ${ }^{6}$ \\ Vera Ehrenstein' \\ 'Department of Clinical Epidemiology, \\ Aarhus University Hospital, Aarhus, \\ Denmark; ${ }^{2}$ Department of Oral \\ and Maxillofacial Surgery, Aarhus \\ University Hospital, Aarhus, Denmark; \\ ${ }^{3}$ Department of Oral and Maxillofacial \\ Surgery, Aalborg University Hospital, \\ Aalborg, Denmark; ${ }^{4}$ Center for \\ Observational Research, Amgen Inc., \\ Thousand Oaks, CA, USA; ${ }^{5}$ Center for \\ Pharmacoepidemiology, Department \\ of Medicine Solna, Karolinska \\ Institutet, Stockholm, Sweden; \\ ${ }^{6}$ Department of Global Public Health \\ and Primary Care, University \\ of Bergen, Bergen, Norway
}

Correspondence: Henrik Gammelager Department of Clinical Epidemiology, Aarhus University Hospital, Olof Palmes Allé 43-45, Aarhus N, Denmark $\mathrm{Tel}+45$ 87I 68245

Fax +45 87। 68063

Email hg@dce.au.dk
Background: Osteonecrosis of the jaw (ONJ) is an adverse effect of drugs that suppress bone turnover - for example, drugs used for the treatment of postmenopausal osteoporosis. The Danish National Registry of Patients (DNRP) is potentially valuable for monitoring ONJ and its prognosis; however, no specific code for ONJ exists in the International Classification of Diseases 10th revision (ICD-10), which is currently used in Denmark. Our aim was to estimate the positive predictive value (PPV) of an algorithm to capture ONJ cases in the DNRP among women with postmenopausal osteoporosis.

Methods: We conducted this cross-sectional validation study in the Central and North Denmark Regions, with approximately 1.8 million inhabitants. In total, 54,956 women with postmenopausal osteoporosis were identified from June 1, 2005 through May 31, 2010. To identify women potentially suffering from ONJ, we applied an algorithm based on ICD-10 codes in the DNRP originating from hospital-based departments of oral and maxillofacial surgery (DOMS). ONJ was adjudicated by chart review and defined by the presence of exposed maxillofacial bone for 8 weeks or more, in the absence of recorded history of craniofacial radiation therapy. We estimated the PPV for the overall algorithm and for each separate ICD-10 code used in the algorithm.

Results: Charts were obtained and reviewed for all 60 women with an ICD-10 code potentially representing ONJ. Nineteen potential ONJ cases were confirmed, corresponding to an overall PPV of 32\% (95\% confidence interval: $20 \%-45 \%$ ).

Conclusion: Among women with postmenopausal osteoporosis, only about one-third of the potential ONJ cases identified by our ICD-10 based algorithm were confirmed by medical chart review, despite the restriction to patients treated at DOMS. To capture true ONJ cases among women with postmenopausal osteoporosis, alternative approaches are needed.

Keywords: bisphosphonate-associated osteonecrosis of the jaw, International Classification of Diseases, osteonecrosis of the jaw, osteoporosis, registries, validity

\section{Introduction}

Osteonecrosis of the jaw (ONJ) is a potentially serious adverse effect of treatment with drugs that suppress bone turnover, especially when given in high-dose regimens to cancer patients. ${ }^{1}$ ONJ can also occur in patients receiving low-dose treatments - for example, among women with postmenopausal osteoporosis. ${ }^{2}$ ONJ is defined by the presence of an exposed maxillofacial bone for at least 8 weeks in patients without a history of prior craniofacial radiation therapy. ONJ severity ranges from asymptomatic exposed bone to severe pain, secondary infection, and/or pathological fractures. ${ }^{2}$ 
Valid ascertainment of ONJ is important for monitoring adverse drug reactions and for research on ONJ prevention, treatment, and prognosis. The Danish National Registry of Patients (DNRP) is a potentially valuable source of such data, as it contains information on all hospital contacts, including all visits to departments of oral and maxillofacial surgery (DOMS), to which patients with ONJ are typically referred in Denmark. Diagnoses in the DNRP are coded using the International Classification of Diseases, 10 th revision (ICD-10). ${ }^{3}$ Unlike the clinical modification of the ICD-10 (ICD-10-CM), the ICD-10 does not contain a specific code for ONJ. ${ }^{3}$

We have previously assessed the positive predictive value (PPV) of an ICD-10-based algorithm to identify ONJ among cancer patients in Denmark and among women with postmenopausal osteoporosis in Sweden. ${ }^{4,5}$ However, PPV depends on the prevalence of the disease in the population where the algorithm is used and may also depend on country-specific coding practices and referral patterns. ${ }^{6} \mathrm{We}$ therefore set out to assess the PPV of the algorithm to capture ONJ among women with postmenopausal osteoporosis in Denmark, using the DNRP.

\section{Methods}

We conducted a cross-sectional validation study among women with postmenopausal osteoporosis in the Central and North Denmark Regions (approximately 1.8 million inhabitants), identified from June 1, 2005 through May 31, 2010. The Danish National Health Service provides tax-supported health care to all Danish residents, with universal access to public hospitals, including DOMS. The unique ten digit civil registration number, assigned to all Danish residents since 1968, permits unambiguous linkage between registries used in the study.

The DNRP includes data from non-psychiatric hospital admissions since 1977 . Since 1995, the registry has also covered all emergency room and outpatient clinic visits. Reportable data include patients' civil registration number, dates of admission and discharge, codes for the treating hospital and department, surgical procedures, and up to 20 discharge diagnoses per visit. Since 1994, diagnoses have been coded using the ICD-10, ${ }^{7,8}$

The Aarhus University Prescription Database includes data on prescriptions dispensed in the outpatient pharmacies of the Central and North Denmark Regions, and includes dispensing date and drug type, coded using the Anatomical Therapeutic Chemical classification. ${ }^{9}$ Through linkage of the Danish Civil Registration System, the DNRP, and the Aarhus University Prescription Database, we identified women with postmenopausal osteoporosis. Postmenopausal women were defined as women aged 55 years or older within the study period. Among those, osteoporosis was defined as any diagnosis of osteoporosis or osteoporotic fracture, or record of a medication used to treat osteoporosis (bisphosphonates, selective estrogen receptor modulators, ipriflavone, strontium ranelate, denosumab, parathyroid hormones, and calcitonin) within a 5-year period before the date of fulfillment of postmenopausal status in the study period (a list of relevant codes is provided in Table S1).

Potential ONJ cases were identified among patients registered at one of the three DOMS in Central and North Denmark Regions, using prespecified ICD-10 codes. ICD-codes were selected by the research team together with experts in ONJ to reflect the local coding practices and definitions of ONJ by the American Association of Oral and Maxillofacial Surgeons and the American Society of Bone and Mineral Research. ${ }^{2}$ One of the following ICD-10 codes was required: idiopathic aseptic necrosis of bone (M87.0); osteonecrosis due to drugs (M87.1); osteonecrosis due to previous trauma (M87.2); other secondary osteonecrosis (M87.3); other osteonecrosis (M87.8); osteonecrosis, unspecified (M87.9); inflammatory conditions of the jaw (K10.2); alveolitis of the jaws (K10.3); or periapical abscess with sinus (K04.6).

Because cancer patients may receive higher dosages of drugs that suppress bone turnover, we used the DNRP to identify history of cancer, which was defined as a cancer diagnosis - with the exception of nonmelanoma skin cancer (ICD-10 codes C00-C97, except C44) - within 5 years before the date of the potential ONJ diagnosis.

One author (HG) obtained and reviewed hospital charts of all potential cases to confirm or rule out ONJ. A confirmed ONJ case was defined by the presence of exposed maxillofacial bone for 8 weeks or longer, with no history of craniofacial radiation therapy evident in the chart. ${ }^{2}$ The additional criterion of current or previous treatment with bisphosphonates was not included, because ONJ in recent years has been found in cancer patients treated with other antiresorptive medications such as the monoclonal antibody, denosumab. ${ }^{10}$ For each confirmed case, we retrieved data from the medical chart regarding bisphosphonate use, dentoalveolar surgery, and denture use before ONJ diagnosis, together with information on the lesion location.

We estimated the PPV as the proportion of confirmed ONJ cases among the potential cases. Furthermore, the PPV was calculated for each ICD-10 code. To examine the potential impact of cancer status, we repeated the analysis among women with postmenopausal osteoporosis without a history of cancer. All estimates were reported with $95 \%$ confidence intervals (CI). 


\section{Results}

During the study period, we identified 60 potential ONJ cases among 54,956 women with postmenopausal osteoporosis in the Central and North Denmark Regions. The number of potential cases sampled using each of the included ICD-10 codes varied from 0 (ICD-10 codes: M87.0, M87.2, M87.3, M87.9, K04.6) to 51 (ICD-10 code K10.2). All medical charts were available for review (retrieval rate, 100\%). The median age of the women with potential ONJ was 72 years (interquartile range: $65-85$ years).

Nineteen out of the 60 potential ONJ cases were confirmed by medical chart review, corresponding to a PPV of $32 \%$ ( $95 \%$ CI: $20 \%-45 \%$ ) (Table 1). Confirmed cases were primarily captured by ICD-10 code K10.2 ( $\mathrm{n}=17)$. The PPVs varied from $0 \%$ for K04.6 (one-sided 97.5\% CI: $0 \%-41 \%$ ) to $100 \%$ for M87.1 (one-sided 97.5\% CI: $25 \%-100 \%$ ) for the different ICD-10 codes included in the algorithm. History of cancer was present among 17 (28\%) of the 60 women with postmenopausal osteoporosis with potential ONJ. After excluding these women, the overall PPV decreased to $21 \%$ (95\% CI: $10 \%-36 \%$ ).

The median age at diagnosis of the 19 patients with confirmed ONJ was 72 years (interquartile range: 67-79). Among the 19 patients, all but one had a record of treatment with bisphosphonates prior to ONJ diagnosis: ten administered intravenously and eight orally. Ten (53\%) patients with confirmed ONJ had a history of cancer. Dentoalveolar surgery had occurred in ten (53\%) of the patients prior to ONJ diagnosis, and twelve patients (63\%) used dentures. The lesions were localized to the mandible in 14 (74\%) patients and to the maxilla in four (21\%) patients. One patient had lesions both in the mandible and maxilla (Table 2).

\section{Discussion}

In this validation study, we found that only about one-third of the potential ONJ cases identified by the algorithm based on ICD-10 codes registered at DOMS corresponded to true ONJ, as confirmed by medical chart review; however, there was large variation in the PPV among the different ICD-10 codes.

The overall PPV of the suggested algorithm to identify ONJ cases was slightly higher than that reported in a previous Danish study evaluating the PPV of the same algorithm among cancer patients in Denmark (PPV 20\% [95\% CI: $12 \%-29 \%]){ }^{4}$ This is unexpected, given that cancer patients receive higher doses of antiresorptive treatment and are expected to have a higher prevalence of ONJ. This finding may be explained by referral practices whereby some cancer patients are routinely referred to DOMS, for example, for a prophylactic oral exam and treatment prior to start of intravenous treatment with antiresorptive medications. NonONJ conditions in those patients could potentially have been coded using one of the ICD-10 codes in the current algorithm. In Sweden, the PPV for the same ONJ algorithm among women with postmenopausal osteoporosis was lower than that reported here (PPV 18\% [95\% CI: 10\%-28\%]). ${ }^{5}$ This may result from differences between countries in referral patterns and in preferences for particular ICD-10 codes in settings where no specific code for ONJ exists.

The estimated PPV of the proposed ICD-10-based ONJ algorithm in Denmark is lower than PPVs typically reported for specific diagnostic codes in the DNRP, usually above $85 \% .^{11-13}$ The primary explanation may be the absence of a specific code for ONJ in the current version of ICD-10 used in Denmark; as a result, the codes in the suggested algorithm are also used for conditions other than ONJ.

Our study has limitations. First, we could not estimate the sensitivity or specificity of the suggested codes, because we did not have access to an independent sample of true ONJ cases, nor do we know the true prevalence of ONJ.

Table I PPV of an algorithm to identify ONJ based on ICD-I0 codes and treatment at departments of oral and maxillofacial surgery, Central and North Denmark Regions, 2005-20I0

\begin{tabular}{|c|c|c|c|c|}
\hline & Total (n) & Confirmed ONJ (n) & Not confirmed ONJ (n) & PPV, \% (95\% Cl) \\
\hline Overalla & 60 & 19 & 41 & $32(20-45)$ \\
\hline Idiopathic aseptic necrosis of bone (M87.0) & 0 & 0 & 0 & \\
\hline Osteonecrosis due to drugs (M87.I) & I & 1 & 0 & $100(25-100)^{b}$ \\
\hline Osteonecrosis due to previous trauma (M87.2) & 0 & 0 & 0 & \\
\hline Other secondary osteonecrosis (M87.3) & 0 & 0 & 0 & \\
\hline Other osteonecrosis (M87.8) & 3 & 2 & I & $67(9-99)$ \\
\hline Osteonecrosis, unspecified (M87.9) & 0 & 0 & 0 & \\
\hline Inflammatory conditions of the jaw (KI0.2) & 51 & 17 & 34 & $33(2 \mid-48)$ \\
\hline Alveolitis of the jaws $(\mathrm{K} \mid 0.3)$ & 2 & 1 & 1 & $50(13-99)$ \\
\hline Periapical abscess with sinus (K04.6) & 7 & 0 & 7 & $0(0-4 I)^{b}$ \\
\hline
\end{tabular}

Notes: aPatients could have more than one ICD-10 code; bone-sided, $97.5 \% \mathrm{Cl}$.

Abbreviations: $\mathrm{Cl}$, confidence interval; ICD, International Classification of Diseases; $\mathrm{n}$, number; ONJ, osteonecrosis of the jaw; PPV, positive predictive value. 
Table 2 Characteristics of the 19 women with postmenopausal osteoporosis with confirmed ONJ, North and Central Denmark Regions, June I, 2005 through May 31, 2010

\begin{tabular}{ll}
\hline Characteristics & Number (\%) \\
\hline Age at ONJ diagnosis, median (IQR) & $72(67-79)$ \\
Malignancies $^{\mathrm{a}}$ & $10(53 \%)$ \\
Use of bisphosphonates $^{\mathrm{b}}$ & $18(95 \%)$ \\
Oral & $8(42 \%)$ \\
$\quad$ Intravenous & $10(53 \%)$ \\
Dentoalveolar surgery & $10(53 \%)$ \\
Use of dentures & $12(63 \%)$ \\
Location of exposed bone & \\
Mandible & $14(74 \%)$ \\
Maxilla & $4(21 \%)$ \\
Both jaws & $1(5 \%)$ \\
\hline
\end{tabular}

Notes: aValues are expressed as number (percentage), unless otherwise indicated; bprior to ONJ occurrence.

Abbreviations: IQR, interquartile range; $\mathrm{ONJ}$, osteonecrosis of the jaw.

This is a common limitation of validation studies of databases. Second, we applied a broad list of ICD-10 codes selected by the research team together with ONJ experts that could be related to ONJ. Some of the suggested ICD-10 codes did not capture any true cases. Nevertheless, the results of this study do not allow for narrowing of the algorithm to particular codes because code-specific PPVs are based on too few observations. Third, the PPV of the suggested ONJ algorithm was evaluated among women with postmenopausal osteoporosis, and results may therefore not be generalizable to other groups of patients. Fourth, the number of potential ONJ cases was small, resulting in imprecise PPV estimates, particularly for those codes which captured a small number of patients.

\section{Conclusion}

Although the suggested algorithm was based on a combination of ICD-10 codes and registration at DOMS, its overall PPV was low, and code-specific PPVs based on a small number of observations do not allow for improvement of the algorithm by narrowing it to particular codes. These approaches could include the implementation of pan-Nordic guidelines for using specific ICD-10 codes or code combinations to designate $\mathrm{ONJ}$, or they could involve the establishment of dedicated ONJ databases.

\section{Acknowledgments}

This study was partially funded by Amgen, Inc, through a contract administered by Aarhus University. Aarhus University subcontracted part of the work related to this project to the Department of Global Public Health and Primary Care, University of Bergen, Bergen, Norway and to the Center of Pharmacoepidemiology at Karolinska Institutet, Stockholm, Sweden.

\section{Disclosure}

HG, CS, SEN, BN, JB, YTL, HK, GST, and VE are salaried employees of their respective institutions. $\mathrm{CC}$ and $\mathrm{FX}$ are full-time employees of Amgen, Inc. The authors report no other conflicts of interest in this work.

\section{References}

1. Silverman SL, Landesberg R. Osteonecrosis of the jaw and the role of bisphosphonates: a critical review. Am J Med. 2009;122(Suppl 2): S33-S45.

2. Ruggiero SL, Dodson TB, Assael LA, Landesberg R, Marx RE, Mehrotra B; for American Association of Oral and Maxillofacial Surgeons. American Association of Oral and Maxillofacial Surgeons position paper on bisphosphonate-related osteonecrosis of the jaws 2009 update. J Oral Maxillofac Surg. 2009;67(Suppl 5):2-12.

3. World Health Organization. International Statistical Classification of Diseases and Related Health Problems 10th Revision [homepage on the Internet]. Available from: http://apps.who.int/classifications/icd10/ browse/2010/en. Accessed May 16, 2013.

4. Gammelager H, Erichsen R, Antonsen S, et al. Positive predictive value of the International Classification of Diseases, 10th revision, codes to identify osteonecrosis of the jaw in patients with cancer. Cancer Epidemiol. 2012;36(4):381-383.

5. Bergdahl J, Jarnbring F, Ehrenstein V, et al. Evaluation of an algorithm ascertaining cases of osteonecrosis of the jaw in the Swedish National Patient Register. Clin Epidemiol. 2013;5:1-7.

6. Rothman KJ. Epidemiology in Clinical Settings. In: Rothman KJ. Epidemiology: An introduction, 1st ed. New York: Oxford University Press; 2002:198-217.

7. Andersen TF, Madsen M, Jørgensen J, Mellemkjoer L, Olsen JH. The Danish National Hospital Register. A valuable source of data for modern health sciences. Dan Med Bull. 1999;46(3):263-268.

8. Lynge E, Sandegaard JL, Rebolj M. The Danish National Patient Register. Scand J Public Health. 2011;39(Suppl 7):30-33.

9. Ehrenstein V, Antonsen S, Pedersen L. Existing data sources for clinical epidemiology: Aarhus University Prescription Database. Clin Epidemiol. 2010;2:273-279.

10. Saad F, Brown JE, Van Poznak C, et al. Incidence, risk factors, and outcomes of osteonecrosis of the jaw: integrated analysis from three blinded active-controlled phase III trials in cancer patients with bone metastases. Ann Oncol. 2012;23(5):1341-1347.

11. Rix TA, Riahi S, Overvad K, Lundbye-Christensen S, Schmidt EB, Joensen AM. Validity of the diagnoses atrial fibrillation and atrial flutter in a Danish patient registry. Scand Cardiovasc J. 2012;46(3): 149-153.

12. Søgaard M, Kornum JB, Schønheyder HC, Thomsen RW. Positive predictive value of the ICD-10 hospital diagnosis of pleural empyema in the Danish National Registry of Patients. Clin Epidemiol. 2011;3: $85-89$.

13. Vestberg K, Thulstrup AM, Sørensen HT, Ottesen P, Sabroe S, Vilstrup H. Data quality of administratively collected hospital discharge data for liver cirrhosis epidemiology. J Med Syst. 1997;21(1):11-20. 


\section{Supplementary table}

Table SI Case algorithm for osteoporosis

Osteoporosis diagnosis (ICD- 10 codes)

- Osteoporosis with pathological fracture (M80x), osteoporosis without pathological fracture $(\mathrm{M} 8 \mathrm{Ix})$, or osteoporosis in diseases classified elsewhere (M82x)

or

Osteoporotic fracture diagnosis (ICD-I 0 codes)

- Fracture of forearm, humerus, spine, pelvis, or femur (ICD-10: S52x, SI2.0, SI2.I, SI2.2, SI2.7, SI2.9, S22.0, S22.I, S32, T08, S42.2, S42.3, S42.4, S42.7, S42.8, and/or S72x)

- Without concurrent Nordic Medico-Statistical Committee code of external causes of injuries indicating major trauma (EUP2-EUP9 and/or EUM2-EUM9)

or

Osteoporosis medications

- Bisphosphonates (ATC: BM05BA, M05BB or Danish treatment code: BWHB40x), selective estrogen receptor modulators (ATC: G03XCx), ipriflavone (ATC: M05BX0I), strontium ranelate (ATC: M05BX03), denosumab (ATC: M05BX04), parathyroid hormones (H05AA), and/or calcitonin H05BA

Abbreviations: ATC, Anatomical Therapeutic Chemical; ICD-10, International Classification of Diseases, I0th revision.

\section{Publish your work in this journal}

Clinical Epidemiology is an international, peer-reviewed, open access journal focusing on disease and drug epidemiology, identification of risk factors and screening procedures to develop optimal preventative initiatives and programs. Specific topics include: diagnosis, prognosis, treatment, screening, prevention, risk factor modification, systematic

Submit your manuscript here: http://www.dovepress.com/clinical-epidemiology-journal
Dovepress

reviews, risk \& safety of medical interventions, epidemiology \& biostatical methods, evaluation of guidelines, translational medicine, health policies \& economic evaluations. The manuscript management system is completely online and includes a very quick and fair peer-review system, which is all easy to use. 